\title{
Adrenal Incidentaloma: Picking out the High-Risk Patients
}

\section{Author \\ Guido Di Dalmazi}

\section{Affiliation}

Division of Endocrinology - Department of Medical and Surgical Sciences, Alma Mater Studiorum University of Bologna, Bologna , Italy

\section{Key words}

adrenal incidentalomas, non-functioning, subclinical hypercortisolism, autonomous cortisol secretion, fractures, osteoporosis, cardiovascular diseases

$\begin{array}{ll}\text { received } & 06.06 .2018 \\ \text { revised } & 02.08 .2018 \\ \text { accepted } & 22.08 .2018\end{array}$

Bibliography

DOI https://doi.org/10.1055/a-0713-0598

Published online: 29.10.2018

Exp Clin Endocrinol Diabetes 2019; 127: 178-184

(c) J. A. Barth Verlag in Georg Thieme Verlag KG Stuttgart . New York

ISSN 0947-7349

\author{
Correspondence \\ Guido Di Dalmazi \\ Division of Endocrinology - Department of Medical and \\ Surgical Sciences \\ Alma Mater Studiorum University of Bologna \\ S. Orsola-Malpighi Hospital \\ Via Massarenti, 9 \\ 40138 Bologna \\ Italy \\ Tel.: + 39/51/214 3009, Fax: + 39/51/2143080 \\ guido.didalmazi@unibo.it
}

\section{ABSTRACT}

Patients with adrenal incidentalomas and autonomous cortisol secretion (ACS) have been recognized as a category at risk of developing cardiovascular and metabolic co-morbidities. Considering the increasing number of patients affected by this condition, it is important to perform an accurate subtyping of the patients, in the light of a tailored treatment. This review aims to identify the phenotypic characteristics of patients with adrenal incidentalomas and ACS who are at risk of developing severe cortisol-related co-morbidities. ACS has been associated with severe alteration of the cardiovascular profile, including hypertension, diabetes, and atherosclerosis. Moreover, ACS has been identified as a contributing factor to the development of cardiovascular events and related mortality. Patients with ACS are also at risk of incident bone fractures, which occur in half of the patients independently of the bone mass density, and infectious diseases. Severity and persistence of hypercortisolism are among the main contributing factors for cardiovascular diseases and bone fractures. Moreover, progression of hypercortisolism over time and altered circadian cortisol rhythm seem to be important additional factors. The role of potential novel biomarkers is also discussed in this review.

\section{Introduction}

The clinical relevance of autonomous cortisol secretion (ACS) in patients with adrenal incidentalomas has been widely recognized in the last years. This condition has usually been defined in patients with alteration of hormonal values, including a combination of elevated cortisol after dexamethasone suppression test, reduced ACTH, or high urinary free cortisol or late-night cortisol, in the absence of a clear phenotype of Cushing's syndrome. Until 2016, different diagnostic criteria have been used in several studies published in the last 20 years, generating difficulties in the interpretation of the co-morbidities associated with mild hypercortisolism [1].
Eventually, the recent guidelines published in 2016 have clarified several aspects of diagnosis and management of patients with adrenal incidentalomas and ACS, pointing toward the central role of dexamethasone suppression test (DST) as a diagnostic tool [1]. ACS is defined by cortisol levels after DST $>1.8 \mu \mathrm{g} / \mathrm{dL}$ (possible) or $>5 \mu \mathrm{g} / \mathrm{dL}$ (confirmed) in patients with adrenal incidentalomas without catabolic signs of Cushing's syndrome. Several studies have clearly shown that a mild and persistent cortisol hypersecretion is associated with harmful clinical consequences. However, several uncertainties remain on the identification of patient at elevated risk who may benefit from targeted treatments. The aim of this review is to evaluate the current evidence on the main clinically-rel- 
evant outcomes associated with ACS, to identify the clinical and biochemical picture of patients at elevated risk of cardiovascular and metabolic diseases.

\section{Clinically Relevant Outcomes in Adrenal Incidentalomas}

\section{Risk of cardiovascular diseases}

During the last 20 years, increasing evidence from cross-sectional studies showed that patients with adrenal tumors associated with ACS have an impaired cardiovascular profile, as highlighted by the high prevalence of several cardiovascular risk factors, mainly hypertension and diabetes. The link between hypertension and mild cortisol excess has been established in several studies, as shown in a recent review of the literature [2]. Even though a precise calculation of the prevalence of hypertension among patient with ACS is cumbersome, due to the low comparability among the different methods of the studies, hypertension is by far the most frequent cortisol-related co-morbidity found in patients with ACS. Indeed, the prevalence of hypertension in patients with ACS is over $60 \%$ in $2 / 3$ of the studies published in the last 15 years [2]. Moreover, patients with ACS seems to be more prone to develop resistant hypertension, as defined by treatment with more than three antihypertensive medications. Studies investigating the prevalence of ACS among hypertensive patients have shown that excessive cortisol secretion may be found in a negligible number of subjects with essential hypertension (1\%) [3]. However, a study analyzing only patients with resistant hypertension showed that $8 \%$ of hypertensive patients had biochemical evidence of cortisol excess, as defined by cortisol after DST $>1.8 \mu \mathrm{g} / \mathrm{dL}$ and two confirmed records of elevated midnight salivary cortisol levels [4]. It is worth mentioning that among 423 patients included in the initial cohort, 112 ( $26.5 \%$ ) had cortisol values after DST $>1.8 \mu \mathrm{g} / \mathrm{dL}$ with or without additional alterations of the hypothalamic pituitary adrenal axis, a condition that it now recognized as possible autonomous cortisol secretion, in keeping with the current guidelines on adrenal incidentalomas [1].

In addition to hypertension, several markers of subclinical atherosclerosis have been studied in patients with ACS. Among them, higher values of intima-media thickness (IMT) and lower flow-mediated dilation (FMD) have been reported in cross-sectional evidence, in patients with ACS, when compared to non-functioning tumors [5-7]. The association between altered IMT and severity of hypercortisolism has been recently investigated in a meta-analysis of 14 studies that characterized the cardiovascular risk of patients with Cushing's syndrome. IMT, FMD, and atherosclerotic plaques were independently associated with severity and duration of cortisol excess, as shown in patients with active disease [8]. Moreover, improvement of IMT was correlated with the median time of disease remission in cured patients, providing indirect evidence on the role of duration of cortisol excess as an important determinant of the development of subclinical atherosclerosis [8]. Instead, the association between markers of subclinical atherosclerosis and ACS is not yet completely understood, as shown by a few studies providing contrasting results. The lack of agreement among the dif- ferent studies is mainly due to the different parameters that were selected as markers of cortisol hypersecretion, like urinary free cortisol or midnight cortisol, which are frequently within the normal range in patients with ACS. Nonetheless, the association between ACS and increased IMT seems to be a relevant issue only in older patients [5]. Several studies shed light on the potential association between altered markers of subclinical atherosclerotic and nonfunctioning adrenal incidentalomas (reviewed in [9]). Even with the limitation due to the cross-sectional design, those studies raise the question whether steroids other than cortisol may have a role in impairing the cardio-metabolic profile of patients with adrenal incidentalomas. Indeed, retrospective evidence showed that patients bearing non-functioning adrenal incidentalomas have a higher incidence of diabetes in a long-term setting [10].

Thrombotic diathesis is a well-known consequence of Cushing's syndrome. Thromboembolic events have been identified as an important causative factor for cardiovascular events, together with hypertension, in up to $20 \%$ of the patients [11]. Even though fewer studies have investigated the hypercoagulable state in patients with ACS, alteration of several parameters (protein C, free protein S, thrombomodulin, and alpha- 1 antitrypsin) resembling that of Cushing's syndrome have been reported in patients with subclinical cortisol hypersecretion [12].

Long-term retrospective studies shed light on the association between cardiovascular events and excessive cortisol production in patients with adrenal incidentalomas. Three independent studies published in 2014 showed that the incidence of myocardial infarction and stroke was indeed higher than that of non-functioning tumors [13-15]. As shown by Cox regression models, the incidence of cardiovascular events was associated with increasing values of cortisol secretion over time, giving feed to the hypothesis that the progression of hypercortisolism may be an important contributing factor for development cardiovascular events [13]. Interestingly, among the potential contributing factors, previous cardiovascular events, absence of lipid lowering treatment, and hypertension were independent contributors to the incidence of cardiovascular events [13]. Similarly, all-cause and cardiovascular-related mortality were also increased in patients with ACS, with respect to non-functioning tumors [13] and to the general population [15]. Therefore, cortisol levels and their progression over time seem to have a relevant role in determining cardiovascular events in patients with adrenal incidentalomas. However, the cortisol-related mechanisms (direct effects of cortisol on cardiovascular system vs indirect actions through the associated co-morbidities) leading to the cardiovascular damage are still not completely understood. It is important to notice that among cardiovascular diseases, the myocardial infarction is more prevalent over stroke in patients with ACS [16]. Recent evidence highlighted that cardiac performance may be altered in patients with hypercortisolism, adding interesting and novel features to the cardiovascular profile of patients with ACS. A recent cross-sectional analysis of a large cohort of patients with incidentalomas revealed that left ventricular mass index, a marker of cardiac hypertrophy associated with hypertension, and pulse wave velocity, a marker of arterial stiffness, were significantly higher in patients with ACS [17]. Moreover, concentric and eccentric left ventricular hypertrophy was more prevalent in patients with ACS. Notably, those alterations occurred independently of the presence 
and duration of hypertension, and the number of hypertensive drugs, which were not different between patients with non-functioning tumors and those with ACS. Those direct and surrogate markers of altered cardiac performance were independently associated with cortisol levels after DST [17].

Another interesting aspect of the relationship between cardiovascular diseases and ACS is related to two distinct features of hypercortisolism, the severity and the progression of cortisol hypersecretion. As previously mentioned, the occurrence of cardiovascular diseases is independently predicted by the increasing severity of hypercortisolism over time [13]. Additionally, all-cause mortality, with cardiovascular diseases as the most frequent cause, was associated with the mean of cortisol after DST during follow-up, uncovering the importance of the persistence of hypercortisolism over time [13]. The role of cortisol as a risk factor for mortality in ACS has been confirmed by the observation that patients belonging to three different classes of increasing severity of hypercortisolism (cortisol after DST $<1.8 \mu \mathrm{g} / \mathrm{dL}$, between 1.8 and $5 \mu \mathrm{g} / \mathrm{dL}$ and $>5 \mu \mathrm{g} / \mathrm{dL}$, respectively) are at parallel increased risk of all-cause and cardiovascular-related mortality [15].

Additionally, the alteration of the circadian cortisol rhythm has been recognized as a potential contributing factor for the development of cardiovascular events. Evidence from population-based studies and targeted investigations revealed that flattening of diurnal cortisol rhythm is associated with increased cardiovascularrelated mortality in the general population and a higher incidence of adverse cardiovascular events in patients undergoing coronary artery bypass graft surgery $[18,19]$. Less is known about the alterations of the circadian cortisol rhythm in patients with ACS. This issue has been investigated in a few studies, providing contrasting results, mainly due to the different definition of ACS, the different sampling conditions (inpatients for serum cortisol vs outpatients for salivary cortisol), and the different assays for cortisol (immunometric assays vs mass spectrometry-based measurements). A recent cross-sectional study by Ceccato et al. provided the results of the analysis of the circadian salivary cortisol measured by mass spectrometry in 106 patients with adrenal incidentalomas [20]. Patients with cortisol levels after DST $>1.8 \mu \mathrm{g} / \mathrm{dL}$ showed a higher area under the curve (AUC) for salivary cortisol in morning measurements and throughout the day, than those with non-functioning tumors. The difference in the AUC was mainly due to significantly increased levels of cortisol in the morning. No difference was detected in evening and late-night cortisol levels between the two groups. Even though this study points toward a higher morning cortisol exposure in patients with cortisol after DST > $1.8 \mu \mathrm{g} / \mathrm{dL}$, no subgroups analysis for morning ACTH levels nor analysis of the association between cortisol exposure and clinical correlates have been performed. Apart from AUC, more informative analyses have been proposed as integrated measurements of circadian rhythmicity, such as cosinor analysys [21], deconvolution parameters and approximate entropy [22]. However, none of those parameters have been applied to the study of adrenal incidentalomas. The role of circadian cortisol rhythm disruption as a potential marker of severity of hypercortisolism in adrenal incidentalomas is still under investigation. It may be speculated that the altered cortisol rhythm may identify a subgroup of patients at higher risk of co-morbidities. Indeed, in a recent proof of concept study, Debono et al. in- vestigated the potential implications of the restoration of a normal circadian cortisol rhythm in patients with incidentalomas, by using a steroidogenesis inhibitor [23]. Among six patients with increased evening cortisol levels, administration of Metyrapone $500 \mathrm{mg}$ at 6 P.M. and $250 \mathrm{mg}$ at 10 P.M. was able to re-assess the cortisol rhythm. This effect was associated with a parallel normalization of IL-6 levels, a known inflammatory marker associated with cardiovascular risk and endothelial disfunction. Larger prospective studies are strongly needed to investigate the clinical impact of such a targeted treatment in a long-term run.

An important, still unresolved, issue is the impact of co-secretion of aldosterone and steroid precursors in patients with ACS and the implication for cardiovascular diseases. A recent study investigating the 24-h urinary output of steroid metabolites by mass spectrometry pointed toward a prevalent glucocorticoid excess in patients with primary aldosteronism in a non-negligible proportion of patients [24]. The co-secretion was highlighted biochemically, by the increased levels of several glucocorticoid precursors in urine samples, and clinically, by the onset of adrenal insufficiency after surgery. In a recent case series, the immunohistochemical analysis of five tumors associated with ACS and concomitant primary aldosteronism revealed that those tumors were mainly composed by zona fasciculata-like cells, with a heterogeneous expression of CYP11B1 and CYP11B2[25]. A recent study investigating the serum steroid profiling by mass spectrometry in patients with different subtypes of Cushing's syndrome showed increased levels of 11-deoxycortisol and 11-deoxycorticosterone in adrenal-dependent hypercortisolism, when compared to controls [26]. Those alterations were not associated with a parallel increase in aldosterone, giving feed to the hypothesis that those steroid precursors may be produced mainly by fasciculata cells. Additionally, an earlier pilot study published in 2015, showed a hyperresponsiveness of several steroid precursors under 1-24 ACTH stimulation in patients with unilateral adrenal adenomas associated with ACS [27]. The potential pejorative impact of the co-secretion of other steroids in association with cortisol on the cardiovascular system of patients with ACS is an open topic and deserves further investigation.

\section{Risk of bone fractures}

Despite the well-known association between steroid excess and bone fracture risk in patients taking exogenous glucocorticoids, a few studies have been performed in patients with endogenous hypercortisolism. Indeed, endogenous and exogenous glucocorticoid-induced osteoporosis represent two different models of diseases, in terms of severity and duration of cortisol exposure. The mechanisms of glucocorticoid-induced osteoporosis are beyond the scopes of this review and are summarized in a recent paper [28].

The appropriateness of the assessment of bone fractures risk in patients with hypercortisolism by evaluation of bone mass density (BMD) has been largely questioned. Indeed, cortisol-related bone fractures have mainly been associated with altered bone quality and occur independently of the BMD [29]. The occurrence of osteoporosis has been described in $28-50 \%$ of patients with overt Cushing's syndrome [30], whereas vertebral fractures have been reported in a variable proportion of patients with hypercortisolism $(21-76 \%)[31,32]$. Such a wide range is mainly due to the study design and the different methods of assessment of vertebral 
fractures (retrospective evaluations vs targeted investigation by radiological images). Notably, the prevalence of asymptomatic vertebral fractures among patients with endogenous hypercortisolism was $48 \%$ [31]. Fewer studies have investigated the association between ACS and bone fractures. According to a recent metanalysis, the prevalence of vertebral fractures has been estimated in $64 \%$ (95\% confidence interval - CI 56-71\%) [29]. Similarly, the incidence of vertebral fractures during follow-up has been recognized as a major issue, occurring in $48 \%$ of the patients [29]. Among other known risk factors, the increased incidence of new vertebral fractures was associated with the presence of ACS (odd ratio 12, $95 \%$ $\mathrm{Cl} 4-37$ ), defined by the presence of at least two alterations among cortisol after DST $>3 \mu \mathrm{g} / \mathrm{dL}$, increased urinary free cortisol levels, and ACTH levels $<10 \mathrm{pg} / \mathrm{ml}$ [33].

Considering the limitations of using the BMD as a predictor of vertebral fractures in patients with Cushing's syndrome, and that the major problem of cortisol-induced osteoporosis is qualitative rather than quantitative, several techniques have been studied to investigate the bone quality in patients with hypercortisolism. Spinal deformity index (SDI) and trabecular bone score (TBS) have been used frequently, because of their feasibility and relative low cost. SDI is a semiquantitative method that integrates number and severity of bone fractures as a surrogate marker of bone quality [34]. TBS is a grey-level texture measurement calculated from the 2-dimensional images of the dual energy X-ray absorptiometry (DEXA) scans at the lumbar level, which gives information about the microarchitecture of the bone [35]. Patients with ACS showed higher SDI than non-functioning adrenal tumors, in cross-sectional and prospective settings $[33,36]$. The application of TBS to the study of patients with adrenal incidentalomas revealed that ACS is characterized by lower TBS values than those of patients with non-functioning adrenal tumors and control subjects. Moreover, TBS was associated with occurrence of vertebral fractures and cortisol levels, independently of other potential contributing factors [37]. Interestingly, TBS was also associated with the incidence of new vertebral fractures over a 2-year period, independently of the BMD [37]. A recent large cross-sectional study has confirmed the superiority of TBS over BMD in the evaluation of bone fragility in patients with various degree of hypercortisolism, including ACS and Cushing's syndrome of different etiologies. Patients with ACS showed lower TBS values $(1.3 \pm 0.1)$, when compared to patients with non-functioning adrenal tumors $(1.4 \pm 0.1)(P<0.04)$, regardless of $B M D[38]$.

Bone fractures in patients with hypercortisolism have been associated with either exposure and duration of cortisol hypersecretion [39]. Even though the excessive cortisol production undoubtedly plays a pivotal role in this context [28], the involvement of additional factors associated with hypercortisolism in increasing the risk of fractures, like sarcopenia, as a potential risk of fall, is still under investigation. Indeed, patients with hypercortisolism are characterized by severely altered muscle mass and performance, which is correlated with severity of hypercortisolism, as shown by radiological and clinical evidence $[40,41]$. Even though the differences were clearer for overt Cushing's syndrome, a reduced muscle mass was also detected in patients with ACS, who do not have clinical stigmata of sarcopenia. A thorough evaluation of muscle performance in patients with ACS is still lacking to date. Recent evidence highlighted that some degrees of altered protein metabo- lism may feature the biochemical phenotype of patients with ACS, with several disturbances of amino acid metabolism, including reduction of histidine, proline, and kynurenine levels [42]. Those alterations have been found in patients with overt Cushing's syndrome and in patients with ACS and were associated with the severity of hypercortisolism [42]. Considering the role of histidine and proline as component of collagen matrix, it may be speculated that their alteration may have a potential impact on muscle strength and sarcopenia. To further support the muscular implication of ACS, a recent report on a small cohort of patients showed that women affected by ACS had a significant reduction in muscle mass than those with non-functioning tumors, as assessed by bioelectrical impedance analysis [43]. Additionally, the role of reduced androgens has been identified as an important determinant of reduced muscle mass, which is a well-known cortisol-related clinical consequence [44]. Several reports have highlighted that patients with either Cushing's syndrome or ACS have lower levels of DHEA and androstenedione than subjects bearing non-functioning tumors and controls, independently of age [26, 27]. The relationship between sarcopenia, androgens, and risk of fall has been highlighted in a very recent large population-based studies, showing that lower DHEA and DHEA-sulphate were associated with increased incidence of falls [45].

A thorough evaluation of muscle performance and its relationship with reduced androgens in patients with ACS deserves further evaluation.

\section{Risk of infectious diseases}

Infectious diseases are a known complication of hypercortisolism. A recent population-based cohort study showed that patients with overt Cushing's syndrome are at increased risk of mortality due to infections, with hazard ratio of 4.9 (95\% Cl 3.7-6.4) [46]. The increased mortality rate was associated with cortisol secretion independently of the specific subtypes of Cushing's syndrome and persisted elevated for several years after treatment [46]. The cortisolrelated mechanisms underlying immune system disruption are still not completely understood. Direct and indirect effects of cortisol, through immunosuppression, vascular damage, and hyperglycemia are among the most frequent causes of susceptibility to infections of patients with Cushing's syndrome. A summary on the molecular mechanisms driving alterations in immune response in hypercortisolism has been recently published elsewhere [11]. Apart from the severity of hypercortisolism, altered circadian cortisol rhythm seems to play a significant role in the alteration of the immune response, mainly by disruption of the circadian lymphocyte rhythm. This concept has been highlighted in a recent randomized controlled trial, showing that restoration of a pseudo-physiological circadian rhythmicity of cortisol improved the immune cell profile of patients with adrenal insufficiency [47]. Specifically, patients receiving modified-release hydrocortisone tabs showed a reduction in the number of pro-inflammatory monocytes CD14(+) CD16(-), lowering their susceptibility to infections [47].

The relevance of infectious complications in patients with ACS has been underestimated and rarely investigated until recently. A long-term retrospective study on the natural history of ACS, previously mentioned, revealed that the infectious complication was the second cause of mortality in patients with ACS, after cardiovascular 


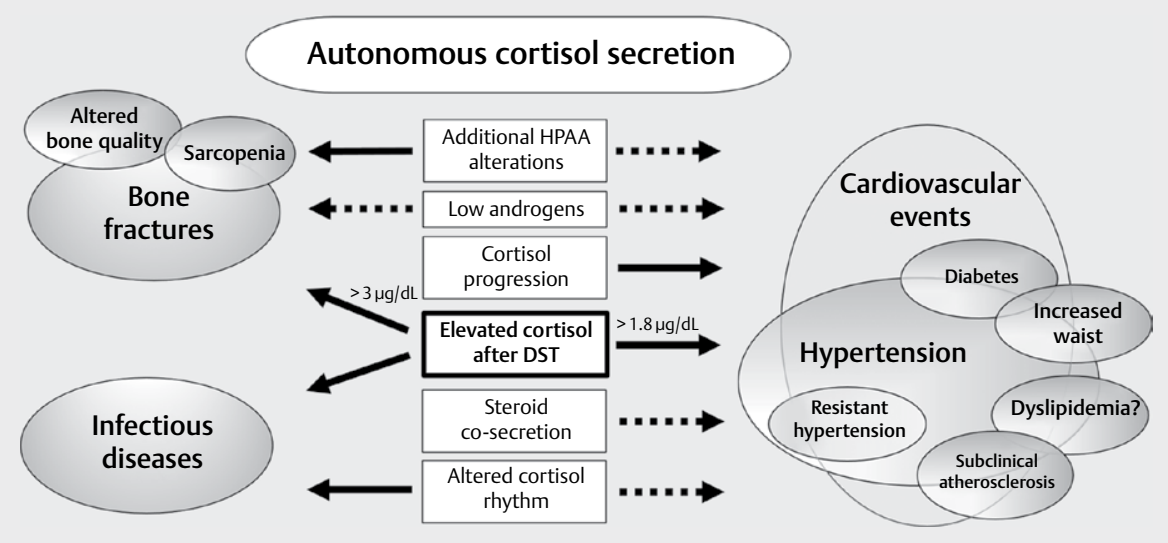

- Fig. 1 Summary of the potential predictive factors for cardiovascular morbidity, bone fractures, and infectious complications associated with autonomous cortisol secretion. Continuous arrows indicate putative mechanisms with evidence in the literature. Dashed arrows indicate potential mechanisms under investigation. HPAA: hypothalamic-pituitary-adrenal axis. DST: dexamethasone suppression test.

diseases [15]. Moreover, the prevalence of infections was higher than that of the general population. This was the first study highlighting the clinical impact of prolonged, although mild, hypercortisolism on the immune system in the long-term run. Moreover, the reduction in IL6 levels after restoration of cortisol rhythm by Metyrapone pointed toward a possible link between altered circadian rhythmicity and infectious complications [23]. Further confirmation is awaited from targeted studies.

\section{The Identification of High-Risk Patients}

A summary of the risk factors for clinical outcomes in patients with ACS is depicted in - Fig. 1. Considering the clinical picture of ACS, it is feasible to speculate about the potential phenotype of patients at risk of developing clinically-relevant outcomes. Cortisol levels after DST $>1.8 \mu \mathrm{g} / \mathrm{dL}$ may be considered as a reliable cut-off to identify a subgroup of patients with adrenal incidentalomas who are at risk of developing cardiovascular events, provided that the measurement is confirmed over time. Moreover, increasing levels of cortisol after DST during subsequent controls should be interpreted as an additional risk factor for cardiovascular diseases. The cortisolrelated risk seems to be an independent contributor to cardiovascular events, mainly in patients with altered cardiovascular profile. The main implication of this hypothesis is that patients with adrenal incidentalomas presenting with hypertension, dyslipidemia, and previous cardiovascular events should be considered for cortisol-lowering treatments, if affected by ACS, or addressed to a careful hormonal follow-up to rule out progression of cortisol secretion, if bearing a non-secreting tumor. Whether co-secretion of additional steroids and altered circadian cortisol rhythm are pejorative factors is unknown to date.

Conversely, the risk of incident bone fractures seems to be relevant in patients with cortisol levels after DST at a higher cut point (i. e. $>3 \mu \mathrm{g} / \mathrm{dL}$ ) and additional hormonal alterations, such as reduced ACTH levels and elevated urinary cortisol. It is feasible that a more severe hormonal profile may be necessary for the develop- ment of bone fragility. Even though additional factors like decreased androgens and sarcopenia seem to contribute to the risk of fractures, stronger evidence is needed. Overall, the occurrence of asymptomatic vertebral fractures has been recorded in half of the patients with ACS. Therefore, a fracture risk assessment should be recommended in all patients with ACS by morphometric evaluation of the spine and assessment of surrogate markers of bone fragility (e.g. SDI or TBS).

The phenotypic characterization of patients at risk of infectious diseases is cumbersome. Given that patients with disrupted circadian rhythm seem to be prone to develop infectious complications, a careful examination of daily cortisol profiles may be useful in all patients with ACS. However, it is important mentioning that the impact of infectious diseases on the well-being of patients with ACS is still under investigation.

\section{Conclusion}

Patients with adrenal incidentalomas are a category at potential risk of life-threatening conditions. Due to the considerable number of patients affected by this condition, a careful characterization of the subtypes of patients at risk is essential to avoid unnecessary examinations in low-risk patients, on one side, and underestimation of the potential co-morbidities in high-risk patients, on the other side. Even though some phenotypic characteristics may be helpful in identifying patients at risk, further studies should be performed to identify specific biomarkers for risk assessment and subtyping of the patients, in the light of a tailored and cost-saving management of patients with adrenal incidentalomas.

\section{Conflict of Interest}

No conflict of interest has been declared by the author. 


\section{References}

[1] Fassnacht M, Arlt W, Bancos I et al. Management of adrenal incidentalomas: European Society of Endocrinology Clinical Practice Guideline in collaboration with the European Network for the Study of Adrenal Tumors. Eur J Endocrinol 2016; 175: G1-G34

[2] Di Dalmazi G, Pasquali R, Beuschlein F et al. Subclinical hypercortisolism: A state, a syndrome, or a disease? Eur J Endocrinol 2015; 173: M61-M71

[3] Omura M, Saito ], Yamaguchi K et al. Prospective study on the prevalence of secondary hypertension among hypertensive patients visiting a general outpatient clinic in Japan. Hypertens Res 2004; 27: 193-202

[4] Martins LC, Conceição FL, Muxfeldt ES et al. Prevalence and associated factors of subclinical hypercortisolism in patients with resistant hypertension. J Hypertens 2012; 30: 967-973

[5] Reimondo G, Allasino B, Coletta M et al. Evaluation of midnight salivary cortisol as a predictor factor for common carotid arteries intima media thickness in patients with clinically inapparent adrenal adenomas. Int J Endocrinol 2015; 2015: 674734

[6] Evran M, Akkus G, Berk Bozdoğan I et al. Carotid intima-media thickness as the cardiometabolic risk indicator in patients with nonfunctional adrenal mass and metabolic syndrome screening. Med Sci Monit 2016; 22: 991-997

[7] Imga NN, Ucar Elalmis O, Muslum Tuna M et al. The relationship between increased epicardial fat thickness and left ventricular hypertrophy and carotid intima-media thickness in patients with nonfunctional adrenal incidentaloma. Int J Endocrinol Metab 2016; 14 : e37635

[8] Lupoli R, Ambrosino P, Tortora A et al. Markers of atherosclerosis in patients with Cushing's syndrome: a meta-analysis of literature studies. Ann Med 2017; 49: 206-216

[9] Di Dalmazi G. Update on the risks of benign adrenocortical incidentalomas. Curr Opin Endocrinol Diabetes Obes 2017; 24: 193-199

[10] Lopez D, Luque-Fernandez MA, Steele A et al. "Nonfunctional" adrenal tumors and the risk for incident diabetes and cardiovascular outcomes: a cohort study. Ann Intern Med 2016; 165: 533-542

[11] Pivonello R, Isidori AM, De Martino MC et al. Complications of Cushing's syndrome: State of the art. Lancet Diabetes Endocrinol 2016; 4: 611-629

[12] Świątkowska-Stodulska R, Sworczak K. Disorders of hemostasis in overt and subclinical hypercortisolism. Exp Clin Endocrinol Diabetes 2013; 121: 588-594

[13] Di Dalmazi G, Vicennati V, Garelli S et al. Cardiovascular events and mortality in patients with adrenal incidentalomas that are either non-secreting or associated with intermediate phenotype or subclinical Cushing's syndrome: A 15-year retrospective study. Lancet Diabetes Endocrinol 2014; 2: 396-405

[14] Morelli V, Reimondo G, Giordano R et al. Long-term follow-up in adrenal incidentalomas: an Italian multicenter study. J Clin Endocrinol Metab 2014; 99: 827-834

[15] Debono M, Bradburn M, Bull M et al. Cortisol as a marker for increased mortality in patients with incidental adrenocortical adenomas. J Clin Endocrinol Metab 2014; 99: 4462-4470

[16] Di Dalmazi G, Vicennati V, Rinaldi E et al. Progressively increased patterns of subclinical cortisol hypersecretion in adrenal incidentalomas differently predict major metabolic and cardiovascular outcomes: A large cross-sectional study. Eur J Endocrinol 2012; 166: 669-677

[17] Sbardella E, Minnetti M, D’Aluisio D et al. Cardiovascular features of possible autonomous cortisol secretion in patients with adrenal incidentalomas. Eur J Endocrinol 2018; 178: 501-511
[18] Kumari M, Shipley M, Stafford M et al. Association of diurnal patterns in salivary cortisol with all-cause and cardiovascular mortality: Findings from the Whitehall II study. J Clin Endocrinol Metab 2011; 96: 1478-1485

[19] Ronaldson A, Kidd T, Poole L et al. Diurnal cortisol rhythm is associated with adverse cardiac events and mortality in coronary artery bypass patients. J Clin Endocrinol Metab 2015; 100: 3676-3682

[20] Ceccato F, Barbot M, Albiger $\mathrm{N}$ et al. Daily salivary cortisol and cortisone rhythm in patients with adrenal incidentaloma. Endocrine 2018; 59: 510-519

[21] Cornelissen G. Cosinor-based rhythmometry. Theor Biol Med Model 2014; $11: 16$

[22] Roelfsema F, van Heemst D, Iranmanesh A et al. Impact of age, sex and body mass index on cortisol secretion in 143 healthy adults. Endocr Connect 2017; 6: 500-509

[23] Debono M, Harrison RF, Chadarevian R et al. Resetting the abnormal circadian cortisol rhythm in adrenal incidentaloma patients with mild autonomous cortisol secretion. J Clin Endocrinol Metab 2017; 102: 3461-3469

[24] Arlt W, Lang K, Sitch AJ et al. Steroid metabolome analysis reveals prevalent glucocorticoid excess in primary aldosteronism. JCl Insight 2017; 2: pii: 93136

[25] Fallo F, Castellano I, Gomez-Sanchez CE et al. Histopathological and genetic characterization of aldosterone-producing adenomas with concurrent subclinical cortisol hypersecretion: A case series. Endocrine 2017; 58: 503-512

[26] Eisenhofer G, Masjkur ], Peitzsch M et al. Plasma steroid metabolome profiling for diagnosis and subtyping patients with cushing syndrome. Clin Chem 2018; 64: 586-596

[27] Di Dalmazi G, Fanelli F, Mezzullo M et al. Steroid profiling by LC-MS/MS in nonsecreting and subclinical cortisol-secreting adrenocortical adenomas. J Clin Endocrinol Metab 2015; 100: 3529-3538

[28] Tóth M, Grossman A. Glucocorticoid-induced osteoporosis: Lessons from Cushing's syndrome. Clin Endocrinol (Oxf) 2013; 79: 1-11

[29] Chiodini I, Vainicher CE, Morelli $V$ et al. mechanisms in endocrinology: Endogenous subclinical hypercortisolism and bone: a clinical review. Eur ] Endocrinol 2016; 175: R265-R282

[30] Mazziotti G, Frara S, Giustina A. Pituitary diseases and bone. Endocr Rev 2018. doi:10.1210/er.2018-00005. [Epub ahead of print]

[31] Tauchmanovà L, Pivonello R, Di Somma C et al. Bone demineralization and vertebral fractures in endogenous cortisol excess: Role of disease etiology and gonadal status. J Clin Endocrinol Metab 2006; 91: 1779-1784

[32] Valassi E, Santos A, Yaneva M et al. The European Registry on Cushing's syndrome: 2-year experience. Baseline demographic and clinical characteristics. Eur J Endocrinol 2011; 165: 383-392

[33] Morelli V, Eller-Vainicher C, Salcuni AS et al. Risk of new vertebral fractures in patients with adrenal incidentaloma with and without subclinical hypercortisolism: A multicenter longitudinal study. J Bone Miner Res 2011; 26: 1816-1821

[34] Genant HK, Delmas PD, Chen P et al. Severity of vertebral fracture reflects deterioration of bone microarchitecture. Osteoporos Int 2007; 18: 69-76

[35] Pothuaud L, Carceller P, Hans D. Correlations between grey-level variations in 2D projection images (TBS) and 3D microarchitecture: Applications in the study of human trabecular bone microarchitecture. Bone 2008; 42: 775-787

[36] Chiodini I, Morelli V, Masserini B et al. Bone mineral density, prevalence of vertebral fractures, and bone quality in patients with adrenal incidentalomas with and without subclinical hypercortisolism: An Italian multicenter study. J Clin Endocrinol Metab 2009; 94: 3207-3214 
[37] Eller-Vainicher C, Morelli V, Ulivieri FM et al. Bone quality, as measured by trabecular bone score in patients with adrenal incidentalomas with and without subclinical hypercortisolism. J Bone Miner Res 2012; 27: 2223-2230

[38] Vinolas H, Grouthier V, Mehsen-Cetre $\mathrm{N}$ et al Assessment of vertebral microarchitecture in overt and mild Cushing's syndrome using trabecular bone score. Clin Endocrinol (Oxf) 2018 [Epub ahead of print]

[39] Vestergaard P, Lindholm J, Jorgensen JO et al. Increased risk of osteoporotic fractures in patients with Cushing's syndrome. Eur J Endocrinol 2002; 146: 51-56

[40] Delivanis DA, Iñiguez-Ariza NM, Zeb MH et al. Impact of hypercortisolism on skeletal muscle mass and adipose tissue mass in patients with adrenal adenomas. Clin Endocrinol (Oxf) 2018; 88: 209-216

[41] Berr CM, Stieg MR, Deutschbein T et al. Persistence of myopathy in Cushing's syndrome: Evaluation of the German Cushing's Registry. Eur J Endocrinol 2017; 176: 737-746

[42] Di Dalmazi G, Quinkler M, Deutschbein T et al. Cortisol-related metabolic alterations assessed by mass spectrometry assay in patients with Cushing's syndrome. Eur J Endocrinol 2017; 177: 227-237
[43] Kim JH, Kwak MK, Ahn SH et al. Alteration in skeletal muscle mass in women with subclinical hypercortisolism. Endocrine 2018 [Epub ahead of print]

[44] Drey M, Berr CM, Reincke M et al. Cushing's syndrome: A model for sarcopenic obesity. Endocrine 2017; 57: 481-485

[45] Ohlsson C, Nethander M, Karlsson MK et al. Serum dhea and its sulfate are associated with incident fall risk in older men: The mros sweden study. J Bone Miner Res 2018 [Epub ahead of print]

[46] Dekkers OM, Horvath-Puho E, Jorgensen JO et al. Multisystem morbidity and mortality in Cushing's syndrome: A cohort study. J Clin Endocrinol Metab 2013; 98: 2277-2284

[47] Isidori AM, Venneri MA, Graziadio C et al. Effect of once-daily, modified-release hydrocortisone versus standard glucocorticoid therapy on metabolism and innate immunity in patients with adrenal insufficiency (DREAM): A single-blind, randomised controlled trial. Lancet Diabetes Endocrinol 2018; 6: 173-185 